\title{
Influência do Nível de Consumo sobre a Degradabilidade das Partículas e Características ligadas à Cinética Ruminal, em Novilhos Pastejando Capim- Elefante
}

\author{
Carlos Augusto de Alencar Fontes ${ }^{1}$, Guilherme Ribeiro Alves ${ }^{2}$, Mário Fonseca Paulino ${ }^{3}$, \\ Eleonora D'Avila Erbesdobler ${ }^{4}$, José Tarcísio Lima Thiebaut ${ }^{5}$, Cláudio Teixeira Lombardi ${ }^{5}$, \\ Hercules Lúcio Gomes ${ }^{6}$
}

\begin{abstract}
RESUMO - Estudou-se a influência do nível de ingestão de pasto sobre a degradabilidade das partículas de capim-elefante nas seções do trato gastrintestinal (TGI) e sobre aspectos ligados à cinética ruminal de bovinos. O delineamento experimental foi o inteiramente casualizado, em parcelas subdivididas, com doze tratamentos nas parcelas (fatorial 2x6, sendo dois níveis de ingestão de pasto: alimentação restrita - $\mathrm{NA}_{1}$ e alimentação irrestrita - $\mathrm{NA}_{2}$ e seis épocas de abate) e as cinco seções do TGI nas subparcelas. Foram usados 36 novilhos, mantidos em regime exclusivo de pasto de capim-elefante. Ao início do experimento e a cada 35 dias, foram abatidos três animais de cada nível alimentar, pesando-se e amostrando-se as digestas das várias seções do TGI. Utilizou-se o peneiramento seco das digestas na determinação do tamanho médio de partículas (TMP) e o módulo de finura (MF). O TMP e o MF da digesta do rúmen-retículo (RR) foram maiores que das demais seções. O nível alimentar não influenciou o TMP. Verificou-se, entretanto, maior MF na digesta do RR, para animais do tratamento $\mathrm{NA}_{2}$. Houve evidência de que partículas menores que $1,19 \mathrm{~mm}$ atravessam preferencialmente o orifício retículoomasal. Não se verificou influência do nível alimentar sobre as taxas de desaparecimento da MS, FDN e FDN digestível no RR, bem como sobre as taxas de passagem da FDN no RR, estimadas em 3,65 e 3,75\%/hora, e suas taxas de degradação, estimadas em 2,46 e 3,12\%/hora, para os tratamentos $\mathrm{NA}_{1}$ e $\mathrm{NA}_{2}$, respectivamente.
\end{abstract}

Palavras-chave: nível alimentar, degradabilidade de partículas, digesta, cinética ruminal, bovinos, capim-elefante

\section{Influence of the Level of Intake on the Degradability of Particles and on Traits of Ruminal Kinetics in Steers Grazing Elephantgrass}

\begin{abstract}
The influence of the level of forage intake on the degradability of particles of elephant grass in the sections of gastrointestinal tract (GIT) of bovines and on some traits of ruminal kinetics was studied. A completely randomized split plot design was used, with twelve treatments in the main plots (a $2 \times 6$ factorial, comprising two levels of feeding: restricted - $\mathrm{NA}_{1}$ and unrestricted - $\mathrm{NA}_{2}$, and six slaughter times) and the five sections GIT in the split plots. Thirty-six steers maintained exclusively under grazing in elephant grass were used. At the beginning of the trial and at each thirty five days, three animals from each feeding level were slaughtered, and the weights and samples of digesta from each GIT section were taken. The dry sieving was used to determine the average particle size (APS) and the modulus of fineness (MF) of digesta. The APS and de MF of digesta from RR were larger than those from the other GIT sections. Feeding level had no influence on APS. However, MF was higher in RR digesta of animals from $\mathrm{NA}_{2}$ treatment. There was evidence that particles smaller than $1.19 \mathrm{~mm}$ leave preferentially the rumen. There was no influence of feeding level on disappearance rates of DM, NDF and indigestible NDF from the rumen, as well as on passage rates of NDF from RR which corresponded to 3.65 and $3.75 \% \mathrm{~h}^{-1}$, and on digestion rates of NDF in the rumen, corresponding to $2.46 \mathrm{e} 3.12 \% \mathrm{~h}^{-1}$, for treatments $\mathrm{NA}_{1}$ and $\mathrm{NA}_{2}$, respectively.
\end{abstract}

Key Words: feeding level, degradability of particles, digesta, ruminal kinetics, bovines, elephantgrass

\section{Introdução}

Altos níveis de consumo de volumosos, em ruminantes, estão associados a elevadas taxas de passagem da digesta no trato gastrintestinal. Entretanto, a degradação microbiana dos carboidratos constituintes da fibra requer tempo de retenção suficientemente longo, de forma a maximizar a produção de energia. O aumento da porção fibrosa das plantas ocasiona aumento do tamanho de partículas, afetando a saída do material do rúmen-retículo (RR) e a concentração dos nutrientes ao longo do trato gastrintestinal (OSBOURN et al., 1976; POPPI et al., 1980).

A quebra das partículas da fibra deve ocorrer até

\footnotetext{
1 Professor - UENF/CCTA - 28015-620 - Campos dos Goytacazes, RJ. E.mail: cafontes@uenf.br

2 Estudante de Doutorado - UENF/CCTA - 28015-620 - Campos dos Goytacazes, RJ. E.mail: guilherm@uenf.br

3 Professor - UFVIDZO.

4 Estudante de Doutorado - UENF/CBB - 28015-620 - Campos dos Goytacazes, RJ. E.mail: lola@uenf.br

5 Técnico de nível superior - UENF/CCTA.

${ }^{6}$ Estudante de graduação - UENF/CCTA.
} 
que atinjam tamanho e densidade que lhes possibilite passar no orifício retículo-omasal. A degradação física das forragens ingeridas pelos ruminantes, em fragmentos cada vez menores, dá-se pela combinação dos eventos: mastigação inicial durante a ingestão; mastigação durante a ruminação; fricção devido à movimentação da digesta e digestão microbiana (FAICHNEY,1986). Estudos de POPPI et al. (1981b) e McLEOD E MINSON (1988a) evidenciaram que a mastigação associada à ruminação tem papel preponderante na redução do tamanho das partículas.

A degradação física, ou redução do tamanho de partícula, é considerada nos modelos americano e australiano descritos, respectivamente, por Mertens et al., em 1984, citados por VAN SOEST et al. (1988) e FAICHNEY (1986). O modelo americano, desenvolvido com vacas leiteiras de alta produção, estabelece o tamanho crítico das partículas para deixarem o rúmen em 3-4 mm, enquanto o modelo australiano postula que o tamanho crítico seria de 1-2 $\mathrm{mm}$. O modelo australiano baseia-se em animais alimentados com forragens, o que pode ser responsável pela divergência dos resultados.

As partículas com maior probabilidade de passarem para o omaso são aquelas localizadas na região ventral do retículo, durante a abertura do orifício retículo-omasal (REID, 1984; WAGHORN et al., 1986). As partículas pequenas e de maior densidade são as que ocorrem em maior concentração nesta região, o que explica seu fluxo preferencial (SUTHERLAND, 1988).

As partículas de alimento e digesta têm sido subdivididas em frações, de acordo com o tamanho, utilizando-se técnicas de peneiramento seco e úmido. A técnica do peneiramento seco, baseada na utilizada para solos e fertilizantes, foi padronizada para alimentos moídos e triturados (ASAE, 1969a,b; ENSOR et al., 1970).

O alimento ingerido pode deixar o rúmen-retículo pela passagem para as seções inferiores do trato gastrintestinal ou por degradação microbiana. Temse demonstrado que a taxa de desaparecimento do material ingerido depende de sua composição química e física, da taxa de degradação microbiana, do tamanho das partículas e do $\mathrm{pH}$ do rúmen (SUTHERLAND, 1988).

Quando o nível de consumo é estável, a taxa de desaparecimento do alimento ou de uma fração alimentar equivale à sua taxa de ingestão. A taxa de ingestão ou desaparecimento tem sido expressa como taxa de ingestão por hora $\left(\mathrm{K}_{\mathrm{i}} \mathrm{h}^{-1}\right)$, que corresponde à ingestão diária de MS da fração considerada, dividido pelo seu conteúdo ruminal e, posteriormente, por 24. A taxa de ingestão ou desaparecimento equivale ao somatório das taxas de passagem $\left(\mathrm{K}_{\mathrm{p}} \mathrm{h}^{-1}\right)$ e de degradação $\left(\mathrm{K}_{\mathrm{d}} \mathrm{h}^{-1}\right)$ (BOSCH e BRUINING, 1995).

Métodos diretos e indiretos têm sido desenvolvidos para quantificar o conteúdo de material do rúmenretículo, as taxas de passagem de suas fases sólida e líquida e a taxa de degradação. As medições diretas do conteúdo ruminal e da taxa de passagem podem ser realizadas fazendo-se a evacuação ruminal e a determinação do fluxo diário de material que deixa o rúmen, utilizando-se animais fistulados no rúmen, no abomaso ou no intestino. Entre os métodos indiretos, tem sido amplamente utilizada a técnica in situ, para determinação de taxas de degradação e a marcação das partículas do alimento com cromo ou terras raras, para estimar as taxas de passagem. Vários autores citados por STENSIG et al. (1998), entretanto, concluíram que a associação da taxa de digestão determinada in situ com a taxa de passagem da fibra, determinada tanto por indicadores quanto por evacuação ruminal, subestima consideravelmente a digestibilidade in vivo.

SHAVER et al. (1988), utilizando os indicadores Yb-EDTA, Sm-EDTA e Cr-EDTA, como marcadores de forragem, concentrado e líquidos, respectivamente, concluíram que os conteúdos de MS e líquidos ruminais foram superestimados em 15,7 e $15,2 \%$, respectivamente, em relação aos valores obtidos por evacuação ruminal e concluíram que o tempo de retenção obtido com base nos indicadores deve ser usado como valor relativo e não como valor absoluto. Por sua vez, STENSIG et al. (1998), utilizando o Itérbio $(\mathrm{Yb})$, concluíram que o indicador subestimou o tempo médio de retenção da FDN, determinado por esvaziamento ruminal. Como conseqüência, a digestibilidade da FDN no rúmen era consideravelmente subestimada, quando a taxa de passagem estimada com base no $\mathrm{Yb}$ era combinada às taxas de digestão.

Um método aproximado para se estudar a cinética ruminal foi descrito por DADO e ALLEN (1995), e posteriormente, utilizado com bons resultados por STENSIG et al. (1998). Por este método, são utilizados animais fistulados apenas no rúmen. O conteúdo ruminal é determinado diretamente, por esvaziamento do rúmen. A produção fecal e a digestibilidade aparente no trato gastrintestinal são medidos utilizan-

Rev. bras. zootec., 30(6S):2134-2144, 2001 
do-se a FDN indigestível (FDNI) como indicador. A taxa de passagem da FDNI do rúmen $\left(\mathrm{K}_{\mathrm{p}} \mathrm{h}^{-1}\right)$ é calculada dividindo-se o consumo médio, por hora, em $\mathrm{kg}$, de FDNI pelo seu conteúdo ruminal, em $\mathrm{kg}$, com base no modelo de primeira ordem de dois compartimentos proposto por WALDO et al. (1972) para digestão de celulose, aplicado para FDN. O teor de FDNI da digesta do rúmen é determinado como o teor de FDN no resíduo indigestível de uma amostra de digesta, após sua incubação in vitro por 120 horas, em meio ruminal tamponado, sem adição de pepsina. O método assume que a taxa de passagem da FDN digestível é igual à da FDNI e que a taxa de ingestão da FDNI é um bom estimador da taxa de passagem, considerando-se que a mesma só pode desaparecer do rúmen por passagem, uma vez que sua digestibilidade seria nula. São também calculadas as taxas de digestão da FDN digestível e a digestibilidade aparente da FDN no rúmen.

Há poucas informações na literatura sobre as características físicas da digesta e da cinética de degradação e de passagem de forrageiras tropicais em bovinos sob pastejo, não tendo sido encontradas referências sobre a influência do nível de ingestão de pasto sobre essas características. O presente trabalho foi conduzido objetivando-se estudar a influência do nível de ingestão de pasto sobre o tamanho médio de partícula e o módulo de finura da digesta, bem como sobre características ligadas à cinética ruminal em novilhos pastejando capim-elefante.

\section{Material e Métodos}

Foram utilizados 36 novilhos 3/4 Gir-Holandês, com idade inicial média de 20 meses e peso vivo médio de $300 \mathrm{~kg}$, os quais foram submetidos a pastejo rotacionado, em capim-elefante, com período de ocupação de 4 dias e descanso de 32 dias e taxa de lotação programada de 6 animais por hectare, no período de outubro a maio de 1997.

Os animais foram divididos, aleatoriamente, em doze grupos, envolvendo a combinação de dois níveis de alimentação e seis épocas de abate. Os níveis de alimentação compreenderam: restrição alimentar, limitando-se o tempo de pastejo a 4 horas por dia (nível de alimentação $\mathrm{NA}_{1}$ ) e sem restrição alimentar, em que os animais permaneceram todo o tempo nas pastagens (nível de alimentação $\mathrm{NA}_{2}$ ). A restrição alimentar por meio de tempo de pastejo teve o objetivo de permitir que os animais dos dois grupos consumissem pasto de qualidade semelhante.
Em cada época de abate, ao início do experimento, após período de adaptação de 15 dias, e a cada 35 dias, foram abatidos três animais do tratamento $\mathrm{NA}_{1}$ e três do $\mathrm{NA}_{2}$. Para se manter a pressão de pastejo constante, os animais abatidos foram substituídos por outros semelhantes.

O consumo alimentar individual foi estimado, nos oito dias que antecederam cada abate, utilizando-se o indicador $\mathrm{Cr}_{2} \mathrm{O}_{3}$ para estimar a excreção fecal e determinando-se a indigestibilidade da extrusa por meio da técnica de digestibilidade in vitro (TILLEY e TERRY, 1963). Para tal, além dos animais experimentais, foram utilizados quatro animais semelhantes, fistulados no esôfago, para obtenção de amostras da extrusa, nos dias de coleta de amostras fecais.

Antes de cada abate, os animais experimentais receberam, durante 16 dias, duas porções de $5 \mathrm{~g}$ de $\mathrm{Cr}_{2} \mathrm{O}_{3}$, acondicionados em cartuchos de papel e colocados diretamente no esôfago, às 10 e $15 \mathrm{~h}$. Nos últimos oito dias, foram coletadas amostras fecais individuais, nos momentos de fornecimento de $\mathrm{Cr}_{2} \mathrm{O}_{3}$. Nesses mesmos dias, os animais fistulados no esôfago foram utilizados para coleta de amostras de extrusa, durante 30 a 40 minutos, após permanecerem em jejum durante 12 horas.

Nos dias de abate, efetuado a partir das $5 \mathrm{~h}$, os animais do grupo $\mathrm{NA}_{2}$ permaneceram no pasto até o momento do abate, enquanto os animais $\mathrm{NA}_{1}$ permaneciam nos currais, a partir das $16 \mathrm{~h}$ do dia anterior, após as quatro horas de pastejo.

Após o abate e evisceração, o trato gastrintestinal (TGI) foi removido e pesado. A seguir, foram amarradas e seccionadas as junções entre os compartimentos: rúmen-retículo e omaso, omaso e abomaso, abomaso e intestino delgado, intestino delgado e intestino grosso. As seções foram então pesadas cheias e após esvaziadas e lavadas, para se determinar o peso de digesta contido em cada uma. O conteúdo de digesta de cada compartimento foi homogeneizado em balde, retirando-se amostras que foram armazenadas em congelador a $-15^{\circ} \mathrm{C}$, para posterior processamento e análise.

As características físicas da digesta foram determinadas, após preparo das amostras de digesta fresca, segundo a técnica descrita por SMITH e WALDO (1969). Posteriormente, foi feito o peneiramento a seco, em equipamento PRODUTEST, com rotor na posição 10 , com tempo de peneiramento de 10 minutos, de acordo com os procedimentos adotados pela AMERICAN DAIRY SCIENCE

Rev. bras. zootec., 30(6S):2134-2144, 2001 
ASSOCIATION (1970). As peneiras utilizadas tinham aberturas de 0,$149 ; 0,297 ; 0,590 ; 1,190 ; 2,380$; e $4,760 \mathrm{~mm}$, com $20 \mathrm{~cm}$ de diâmetro e $5 \mathrm{~cm}$ de profundidade. O peso seco retido em cada peneira foi expresso em porcentagem do peso seco total retido nas seis peneiras, enquanto o peso seco acumulado para cada peneira correspondeu à proporção de peso seco total retido por uma peneira, mais aquele retido nas peneiras de tamanho do poro imediatamente superiores, expresso em porcentagem. Além disso, foi determinada a proporção de partículas que passou em cada peneira, incluindo-se nos cálculos o peso de matéria que passou pela peneira de menor abertura.

O tamanho médio de partícula(TMP), emmilímetros, da digesta das seções de TGI, de cada animal, foi calculado por intermédio de parâmetros da equação exponencial sugerida por Pond et al. (1984), citados por FISCHER et al. (1988):

$$
\mathrm{R}=100 \mathrm{e}^{-\mathrm{K}(\mathrm{S}-\mathrm{W})}
$$

em que: $\mathrm{R}$ corresponde à porcentagem acumulada do peso seco da amostra por peneira; $\mathrm{S}$, ao tamanho da abertura do poro da peneira $(\mathrm{mm}) ; \mathrm{W}$, ao menor tamanho de partícula (mm); e K, ao declínio constante da curva exponencial, que indica a proporção de partículas acima de algum tamanho de tela que passará por uma peneira mais larga.

Os valores de W e K são estimados, ajustando-se a equação, a partir dos valores de $\mathrm{R}$ e dos valores de $\mathrm{S}$ correspondentes.

No ajuste do modelo, foi utilizado o algoritmo Quasi-Newton, do procedimento Nonlinear Estimation do programa STATISTICA versão 5.0. Como premissa do programa, fornece-se uma estimativa inicial do valor de W. A partir daí, o programa ajusta a equação, chegando aos valores reais de K e W.

Conhecendo-se os valores de K e W, o TMP é determinado utilizando-se a fórmula:

$$
\mathrm{TMP}=1 / \mathrm{K}+\mathrm{W}
$$

O módulo de finura (MF) é uma medida adimensional que expressa a finura da amostra. Foi determinado de acordo com a metodologia recomendada pela AMERICAN SOCIETY OF AGRICULTURAL ENGINEERS (1969a,b) e adotada pela AMERICAN SOCIETY OF ANIMAL SCIENCE (1969).

Utilizou-se um método aproximado, baseado no descrito por DADO e ALLEN (1995) e posteriormente utilizado, com bons resultados, por STENSIG et al. (1998) na determinação de variáveis ligadas à cinética do rúmen-retículo.
Os pesos de digesta do rúmen, obtidos por ocasião do abate dos 36 animais experimentais, nos cinco períodos consecutivos de pastejo, foram tomados como representativos do peso médio diário da digesta ruminal. As médias individuais de consumo alimentar e digestibilidade da FDN, bem como a composição média da extrusa, obtidos nos últimos oito dias que antecederam os abates, foram utilizados no cálculo da ingestão média diária das diferentes frações do alimento.

As taxas de desaparecimento da MS, FDN total e FDN digestível foram consideradas iguais à taxa de ingestão $\left(\mathrm{K}_{\mathrm{i}} \mathrm{h}^{-1}\right)$ (DADO e ALLEN, 1995; STENSIG, 1998), sendo estimadas utilizando-se a fórmula:

$$
\mathrm{K}_{\mathrm{i}}\left(\mathrm{h}^{-1}\right)=\frac{\text { Consumo diário do componente }(\mathrm{kg})}{\text { Conteúdo ruminal do componente }(\mathrm{kg})} / 24
$$

As taxas de passagem da FDN total e digestível, consideradas iguais a da FDNI, foram dadas pela taxa de ingestão da FDNI, uma vez que a mesma só pode deixar o rúmen por meio dos mecanismos de passagem, foram calculadas pela fórmula:

$$
\mathrm{K}_{\mathrm{p}_{\mathrm{FDNI}}}\left(\mathrm{h}^{-1}\right)=\frac{\text { Consumo diário de FDNI }(\mathrm{kg})}{\text { Conteúdo ruminal de FDNI }(\mathrm{kg})} / 24
$$

$\mathrm{O}$ teor de FDNI na digesta do RR foi determinado como o teor de FDN no resíduo indigestível de amostras de digesta, após sua incubação in vitro por 120 horas, em meio ruminal tamponado, sem adição de pepsina, conforme DADO e ALLEN (1995).

A taxa de degradação da FDN digestível foi dada pela diferença entre sua taxa fracionária de desaparecimento e a taxa de passagem:

$$
\mathrm{K}_{\mathrm{dFDND}} \mathrm{h}^{-1}=\mathrm{K}_{\mathrm{i}} \mathrm{h}^{-1}-\mathrm{K}_{\mathrm{p}} \mathrm{h}^{-1}
$$

A digestibilidade da FDN no rúmen (\% FDND rúmen) foi calculada utilizando-se a fórmula:

$\%$ FDND rúmen $=$ Fração de FDN digestível (\%) $\mathrm{x}\left[\mathrm{K}_{\mathrm{d}} /\left(\mathrm{K}_{\mathrm{d}}+\mathrm{K}_{\mathrm{p}}\right)\right]$

O tempo médio de retenção da FDN no rúmen foi calculado como o inverso da taxa de passagem. O tempo de renovação do rúmen (TR) foi calculado pela fórmula:

$$
\mathrm{TR}=\frac{\text { Conteúdo de MS ruminal }(\mathrm{kg})}{\text { Consumo de MS por hora }(\mathrm{kg})}
$$

As análises estatísticas referentes ao TMP e MF da digesta das várias seções do trato gastrintestinal, foram realizadas segundo o esquema em parcelas subdivididas, com 12 tratamentos nas parcelas $\left(\mathrm{I}_{1} \mathrm{~A}_{1}\right.$, $\mathrm{I}_{1} \mathrm{~A}_{2}, \ldots, \mathrm{I}_{2} \mathrm{~A}_{6}$ ), que constituíram um fatorial $2 * 6$, sendo dois níveis de ingestão (I) de volumoso (restrito e não restrito) e seis épocas de abate (A), ao início do 
experimento e após os cinco períodos de pastejo de 35 dias. As cinco seções do trato gastrintestinal constituíram os tratamentos das subparcelas $\left(\mathrm{S}_{1}, \ldots, \mathrm{S}_{5}\right)$. Foram utilizados três animais (An) por época e nível de ingestão, conforme o modelo abaixo:

$\mathrm{Y}_{\mathrm{ijkl}}=\mu+\mathrm{I}_{\mathrm{i}}+\mathrm{A}_{\mathrm{j}}+\mathrm{IA}_{\mathrm{ij}}+\mathrm{E}_{(\mathrm{ijj})}+\mathrm{S}_{\mathrm{k}}+\mathrm{IS}_{\mathrm{ik}}+\mathrm{AS}_{\mathrm{jk}}+$ em que: $Y_{i j k l}=$ observação referente ao animal 1, do nível de ingestão de volumoso $\mathrm{i}$, da época de abate $\mathrm{j}$ e da seção do TGI k; $\mu$ = média geral; $\mathrm{I}_{\mathrm{i}}=$ efeito do nível de ingestão de volumoso $\mathrm{i}$, sendo $\mathrm{i}=1$ restrito $\mathrm{e}$ 2 irrestrito; $A_{j}=$ efeito da época de abate $j$, sendo $j=$ $1,2,3,4,5$ e $6 ; \mathrm{IA}_{\mathrm{ij}}=$ efeito da interação entre nível de ingestão de volumoso i e época de abate $\mathrm{j}$; $\mathrm{E}_{(\mathrm{ij}) 1}=$ erro aleatório associado à parcela, suposto normal e independentemente distribuído, com média zero e variância $\sigma^{2} ; \mathrm{S}_{\mathrm{k}}=$ efeito da seção do TGI $\mathrm{k}$, sendo $\mathrm{k}=1,2,3,4$ e 5; $\mathrm{IS}_{\mathrm{ik}}=$ efeito da interação entre o nível de ingestão de volumoso i e a seção do TGI k; $\mathrm{AS}_{\mathrm{jk}}=$ efeito da interação entre a época $\mathrm{j}$ e a seção

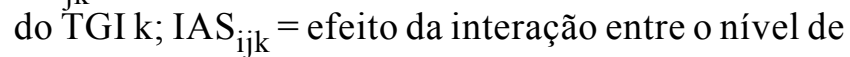
ingestão de volumoso i, época k e seção do TGI k; $\mathrm{E}_{\mathrm{ijkl}}=$ erro associado à subparcela, suposto normal e independentemente distribuído, com média zero e variância $\sigma^{2}$.

Para a análise dos dados referentes às variáveis ligadas à cinética do rúmen-retículo foiutilizado o modelo:

$$
\mathrm{Y}_{\mathrm{ijk}}=\mu+\mathrm{C}_{\mathrm{i}}+\mathrm{N}_{\mathrm{j}}+\mathrm{CN}_{\mathrm{ij}}+\mathrm{e}_{\mathrm{ijk}}
$$

em que: $Y_{i j k}=$ observação referente ao animal $\mathrm{k}$, no período de pastejo $\mathrm{i}$, submetido ao nível nutricional $\mathrm{j}$; $\mu=$ média geral; $C_{i}=$ efeito do período de pastejo i, em que $\mathrm{i}=1,2,3,4$ e $5 ; \mathrm{N}_{\mathrm{j}}=$ efeito do nível de oferta $\mathrm{j}$, sendo $\mathrm{j}=1$ restrito e 2 irrestrito; $\mathrm{CN}_{\mathrm{ik}}=$ efeito da interação entre o período de pastejo i e o nível de oferta $\mathrm{j} ; \mathrm{e}_{\mathrm{ijkl}}=$ erro aleatório, associado a cada observação, pressuposto normal e independentemente distribuído, com média zero e variância $\sigma^{2}$.

\section{Resultados e Discussão}

O consumo alimentar médio \pm erro-padrão dos animais dos dois tratamentos referente aos oito dias que antecederam ao abate, nos cinco períodos de pastejo, foi de $5,41 \pm 0,25$ e 7,99 $\pm 0,25 \mathrm{~kg}$ de MS para os tratamentos com restrição alimentar $\left(\mathrm{NA}_{1}\right)$ e sem restrição alimentar $\left(\mathrm{NA}_{2}\right)$, respectivamente.

A análise de variância dos dados referentes a tamanho médio de partícula (TMP) revelou efeito de interação $(\mathrm{P}<0,05)$ entre o nível de ingestão de forragem e a seção do TGI. Avaliando-se o efeito do nível de ingestão alimentar dentro de seção do TGI, verificou-se diferença apenas no abomaso (AB), tendo ocorrido maior TMP $(\mathrm{P}<0,05)$ em animais submetidos à restrição alimentar (Tabela 1). Os animais submetidos aos dois níveis nutricionais apresentaram TMP semelhante no RR. Provavelmente, a maior ingestão alimentar dos animais do tratamento $\mathrm{NA}_{2}$ foi compensada pela maior atividade de ruminação que ocorre em animais com maior conteúdo ruminal, conforme salientado por DADO e ALLEN (1995), resultando em ausência de diferença no TMP.

Nas comparações entre seções dentro de nível de alimentação, verificou-se maior TMP na digesta do RR que nas demais seções $(\mathrm{P}<0,05)$, nos dois níveis de alimentação. Em ambos os níveis de alimentação, o TMP da digesta do AB foi maior $(\mathrm{P}<0,05)$ que das digestas do intestino delgado (ID) e intestino grosso (IG), sendo também maior $(\mathrm{P}<0,05)$ que da digesta do omaso $(\mathrm{OM})$ nos animais submetidos à restrição alimentar.

O TMP do RR é fortemente afetado pela mastigação inicial durante a ingestão do alimento e, especialmente, pela mastigação durante a ruminação. A mastigação durante a ingestão é um processo bastante eficiente para redução do tamanho de partícula em forragens verdes. MARTZ e BELYEA

Tabela 1 - Tamanho médio de partícula (TMP, mm) na digesta, ao longo do trato gastrintestinal de novilhos, submetidos a dois níveis de alimentação: $\mathrm{NA}_{1}$ - com restrição alimentar; $\mathrm{NA}_{2}$ sem restrição alimentar

Table 1 - Average particle size (APS) in digesta throughout the gastrintestinal tract of steers submitted to two levels of feeding: $N A_{1}$ - restricted fed; $N A_{2}$ - unrestricted fed

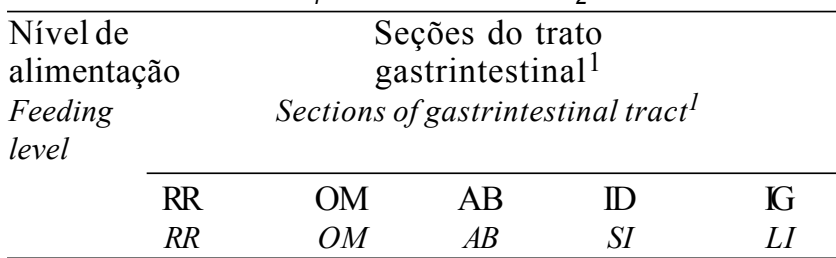

NA $1 \quad 0,9251^{\mathrm{Aa}} \quad 0,5854^{\mathrm{Ac}} \quad 0,7559^{\mathrm{Ab}} \quad 0,5560^{\mathrm{Ac}} \quad 0,5263^{\mathrm{Ac}}$

NA $20,9650^{\mathrm{Aa}} 0,5969^{\mathrm{Abc}} 0,6522^{\mathrm{Bb}} 0,5388^{\mathrm{Ac}} \quad 0,5381^{\mathrm{Ac}}$

${ }^{1} \mathrm{RR}$ - rúmen-retículo; OM - omaso; $A B$ - abomaso; ID - intestino delgado; IG - intestino grosso.

Médias seguidas das mesmas letras maiúsculas na coluna não diferem, segundo o teste $F(P>0,05)$; as médias seguidas das mesmas letras minúsculas na linha não diferem, segundo o teste Tukey $(P>0,05)$.

${ }^{1} R R$ - reticulum-rumen; OM - omasum; $A B$ - abomasum; SI - small intestine; $L I$ - large intestine.

Means followed by the same capital letters in the columns do not differ, by $F$ test $(P>$.05); means followed by the same small letterin the rows do not differ by Tukey test $(P>.05)$. 
(1986) sugeriram que a forragem é reduzida a tamanhos de 1 a $2 \mathrm{~mm}$, antes de ser deglutida. FISHER et al. (1988), usando os dados de Pond et al. (1984), encontraram o valor de $1,96 \mathrm{~mm}$ para TMP da ingesta ruminal, para gramíneas tropicais.

Vários autores citados por SANCHEZ REYES (1994) mostraram que fatores como peso corporal de bovinos, entre 261 e $861 \mathrm{~kg}$, bem como nível de ingestão de matéria seca, não afetaram a distribuição nem o TMP ao longo do TGI, enquanto a freqüência de alimentação afetou tais variáveis. No presente trabalho, o nível de alimentação só influenciou o TMP da digesta do abomaso.

Os valores do TMP da digesta ao longo do TGI verificados no presente trabalho foram inferiores aos encontrados por QUEIROZ et al. (1996a), mas superiores aos observados por FREITAS (1997). Entretanto, QUEIROZ et al. (1996a) alimentaram os animais com ração contendo 50\% de concentrado e 50\% de feno de capim-braquiária, colhido em estádio vegetativo avançado. Por sua vez FREITAS (1997) utilizou feno de coast-cross moído. Segundo ULYATT et al. (1986), forragens verdes ou de melhor qualidade (conforme o presente trabalho) são mastigadas de forma mais eficiente que forragens secas ou de qualidade inferior.

Os valores de TMP do rúmen encontrados no presente trabalho aproximaram-se dos verificados por ALMEIDA (1997), em amostras de digesta coletadas no rúmen de bovinos 24 horas após voltarem a pastejar área contendo várias espécies de forrageiras tropicais, os quais haviam sido, previamente, submetidos a um período de jejum de 16 horas. Em amostras de digesta coletadas em intervalos próximos ao fim do período de jejum, o citado autor observou maiores valores de TMP.

Os resultados apresentados por QUEIROZ et al. (1996a) e LEE e PEARCE (1984) permitem a dedução de uma degradação contínua e significativa das partículas ao longo do TGI, enquanto FREITAS (1997), ULYATT et al. (1986), e POPPI et al. (1980), relatam não haver mudança significativa no TMP da digesta, após a saída do RR. No presente estudo, verificou-se tamanho médio de partícula nitidamente maior no RR que nas demais seções. Além disso, o TMP do AB tendeu a ser ligeiramente superior aos do OM, ID e IG, o que, aparentemente, ocorreu em função de maior dificuldade de dispersão do material, durante o peneiramento e presença de algumas sementes de gramínea invasora (braquiária) na digesta dessa seção, especialmente no primeiro período de pastejo. As sementes observadas na digesta do $\mathrm{AB}$ foram retidas na peneira de $1,19 \mathrm{~mm}$, não acontecendo o mesmo na digesta do RR e das demais seções. Possivelmente, a gravidade específica das sementes permitiu-lhes passar no orifício retículoomasal, embora com tamanho superior a 1,19 mm, e, posteriormente, foram degradadas sob a ação de enzimas e acidez do abomaso, não aparecendo na digesta do ID e IG.

Verificou-se efeito de interação $(\mathrm{P}<0,05)$ entre nível de ingestão de pasto e seção do TGI para a variável módulo de finura (MF). Dessa forma, estudou-se a influência do nível de ingestão de pasto dentro de seção do TGI e comparou-se o MF das digesta das seções dentro de nível de ingestão de pasto (Tabela 2).

O nível alimentar exerceu influência sobre o MF das digestas do RR e AB, verificando-se para animais com maior ingestão alimentar maior MF na digesta do $\mathrm{RR}(\mathrm{P}<0,05)$ e menor na digesta do AB $(\mathrm{P}<0,05)$.

Entre seções dentro de nível alimentar, observouse maior $(\mathrm{P}<0,05)$ módulo de finura no $\mathrm{RR}$ que nas demais seções, nos dois níveis de alimentação, maior no $\mathrm{AB}$ que no $\mathrm{OM}$, em animais submetidos à restrição alimentar, $(\mathrm{P}<0,05)$ e menores valores $(\mathrm{P}<0,05)$ no ID e IG que no $\mathrm{AB}$, para os dois níveis de alimentação.

Tabela 2 - Módulo de finura (MF) na digesta ao longo do trato gastrintestinal de novilhos, submetidos a dois níveis de alimentação: $\mathrm{NA}_{1}$ - com restrição alimentar; $\mathrm{NA}_{2}$ - sem restrição alimentar

Table 2 - Modulus of fineness (MF) of digesta throughout the gastrintestinal tract of steers submitted to two levels of feeding: $N A_{1}$ - restricted fed; $N A_{2}$ - unrestricted fed

\begin{tabular}{lccccc}
\hline $\begin{array}{l}\text { Nível de } \\
\text { alimentação } \\
\text { Feeding }\end{array}$ & \multicolumn{5}{c}{$\begin{array}{c}\text { Seções do trato } \\
\text { gastrintestinal }\end{array}$} \\
level & Sections of gastrintestinal tract & \\
& RR & $\mathrm{OM}$ & $\mathrm{AB}$ & $\mathrm{ID}$ & $\mathrm{IG}$ \\
& $R R$ & $O M$ & $A B$ & $S I$ & $L I$ \\
\hline NA 1 & $2,84^{\mathrm{Ba}}$ & $2,16^{\mathrm{Ac}}$ & $2,48^{\mathrm{Ab}}$ & $2,06^{\mathrm{Acd}}$ & $1,91^{\mathrm{Ad}}$ \\
NA 2 & $3,01^{\mathrm{Aa}}$ & $2,20^{\mathrm{Abc}}$ & $2,25^{\mathrm{Bb}}$ & $2,00^{\mathrm{Acd}}$ & $1,93^{\mathrm{Ad}}$ \\
\hline
\end{tabular}

${ }^{1} \mathrm{RR}$ - rúmen-retículo; OM - omaso; $\mathrm{AB}$ - abomaso; ID - intestino delgado; IG - intestino grosso.

Médias seguidas das mesmas letras maiúsculas na coluna não diferem, segundo o teste $F(P>0,05)$; as médias seguidas das mesmas letras minúsculas na linha não diferem, segundo o teste Tukey $(P>0,05)$.

${ }^{1} R R$ - reticulum-rumen; OM - omasum; $A B$ - abomasum; SI - small intestine; $L I$ - large intestine.

Means followed by the same capital letters in the columns do not differ, by $F$ test $(P>.05)$; means followed by the same small letter in the rows do not differ by Tukey test $(P>.05)$. 
Os valores do MF das digestas das várias seções do TGI foram inferiores aos encontrados por QUEIROZ et al. (1996), que verificaram, em animais de quatro grupos genéticos, MF da digesta ruminal entre 3,84 e 3,91 e degradação contínua das partículas ao longo das seções do TGI. Foram, entretanto, superiores aos valores de 2,35 a 2,47 verificados por FREITAS (1997), na digesta do rúmen, para rações contendo níveis crescentes de concentrado, que também observou maior módulo de finura na digesta do RR que nas demais seções.

Valores de MF da digesta do RR próximos aos do presente trabalho foram relatados por ALMEIDA (1997), que, ao estudar os efeitos de épocas (verão e inverno) e tempos de coleta sobre o MF na digesta do rúmen de novilhos em pasto natural, encontrou valores de 3,44 e 2,75, para coletas efetuadas 4 e 24 horas, respectivamente, após início de pastejo, depois de período de jejum 12 horas, no período de verão, e de 2,98 e 2,76, no período de inverno.

Também POPPI et al. (1985), verificaram MF de 2,96 e 2,93 na digesta ruminal de animais alimentados, respectivamente, com folhas e caules de Digitaria decumbens.

LUNGINBUHL et al. (1990) observaram redução do tamanho das partículas do rúmen, com o decorrer do tempo após a alimentação. Esse efeito foi também verificado por MARTZ e BELYEA (1986), que o atribuíram ao maior tempo existente para a atividade de mastigação. No presente trabalho, os animais submetidos à restrição de pastejo permaneceram cerca de 14 horas em jejum antes do abate, uma vez que eram mantidos diariamente no curral, $16 \mathrm{~h}$ até a manhã seguinte, enquanto aqueles não submetidos à restrição de pastejo, permaneceram no pasto até o momento do abate. A ausência de jejum prévio ao abate pode explicar o maior MF das partículas do rúmen nos animais não submetidos à restrição de pastejo.

Conforme mencionado, o cálculo do TMP baseia-se na porcentagem acumulada de partículas retidas em peneiras de tamanho de malha decrescentes, enquanto no cálculo do MF utilizam-se as porcentagens de partículas retidas em cada peneira (não acumuladas). Em ambos, desconhece-se a porção de partículas que atravessa a menor peneira (de $0,149 \mathrm{~mm}$ ), por se admitir que, mesmo as partículas retidas na peneira de $0,149 \mathrm{~mm}$ não encontram resistência para deixarem o RR (POPPI et al., 1980). Entretanto, no presente trabalho, verificou-se que na digesta do RR, em que é maior a presença de partículas de maior tamanho, pode haver menor proporção de partículas retidas em peneiras intermediárias que na digesta das outras seções do TGI, nas quais o TMP é menor. Além disso, conforme referido anteriormente, houve dificuldade em se conseguir completa desagregação da digesta do $\mathrm{AB}$, de algumas amostras. Por este motivo, a digesta da citada seção apresentou comportamento médio um pouco diferente das digestas do OM, ID e IG.

Os fatos acima referidos ocasionaram valores negativos, quando se procurou calcular a resistência relativa à passagem, das partículas retidas nas várias peneiras, com base nas informações do RR e AB, seguindo a metodologia sugerida por POPPI et al. (1980). Decidiu-se, por isso, avaliar a distribuição dos tamanhos das partículas de toda a amostra da digesta das várias seções, incluindo-se nos cálculos o resíduo que atravessou a peneira de $0,149 \mathrm{~mm}$, de forma a possibilitar inferências sobre a existência de um tamanho crítico de partícula para deixar o RR. Desta forma, foi calculada, para cada seção, a proporção da digesta total que passou em cada peneira. Os resultados são apresentados no Tabela 3.

As informações da Tabela 3 mostram que 86,23 e $84,09 \%$ da digesta do RR (em peso) de animais dos tratamentos $\mathrm{NA}_{1}$ e $\mathrm{NA}_{2}$, respectivamente, foram capazes de atravessar a peneira de $4,76 \mathrm{~mm}$ e 83,35 e $80,15 \%$ atravessaram a peneira de $2,38 \mathrm{~mm}$. Maior resistência à passagem ocorreu a partir da peneira de $1,19 \mathrm{~mm}$, quando cerca de 22,29 e $26,65 \%$ da digesta do RR de animais dos tratamentos $\mathrm{NA}_{1}$ e $\mathrm{NA}_{2}$, respectivamente foram retidos (passaram $77,71 \mathrm{e}$ $73,35 \%$ do material, respectivamente).

Nos animais dos dois tratamentos, as digestas do OM, ID e IG comportaram-se, de modo geral, de forma semelhante. Quase a totalidade da digesta das três seções (assim como do $\mathrm{AB}$ ) passou pela peneira de 2,38 $\mathrm{mm}$. Na peneira de $1,19 \mathrm{~mm}$, houve retenção de menos de $10 \%$ do total de digesta do OM, ID e IG, nos animais de ambos tratamentos, e retenção de cerca de 16,28 e $12,42 \%$ da digesta do $\mathrm{AB}$, para os tratamentos $\mathrm{NA}_{1}$ e $\mathrm{NA}_{2}$, respectivamente. A partir desse ponto, a cada diminuição da malha, passou a ocorrer redução mais pronunciada da quantidade de digesta que atravessou a peneira, em ambos tratamentos.

O fato de mais de $90 \%$ das digestas do OM, ID e IG terem tamanho inferior a $1,19 \mathrm{~mm}$ dá forte indicação de que partículas com tamanho inferior a $1,19 \mathrm{~mm}$

Rev. bras. zootec., 30(6S):2134-2144, 2001 
Tabela 3 - Proporção de partículas menores que o tamanho da abertura de cada peneira, na digesta das várias seções ${ }^{1}$ do trato gastrintestinal, em novilhos submetidos a dois níveis de alimentação: $N A_{1}$ - com restrição alimentar; $N A_{2}-$ sem restrição alimentar

Table 3 - Proportion of particle of digesta from the different section of gastrintestinal tract smaller than the size of each screen, in steers submitted to two levels of feeding: $N A_{1}$ - restricted fed; $N A_{2}$ - unrestricted fed

\begin{tabular}{|c|c|c|c|c|c|c|c|c|c|c|}
\hline \multirow{4}{*}{$\begin{array}{l}\text { Peneira } \\
\text { Screen } \\
(\mathrm{mm})\end{array}$} & \multicolumn{9}{|c|}{$\begin{array}{l}\text { Proporções de partículas (\%) que passaram nas diferentes peneiras } \\
\text { Proportions of particles (\%) which passed through the different screens }\end{array}$} & \\
\hline & \multicolumn{5}{|c|}{$\mathrm{NA}_{1}$} & \multicolumn{5}{|c|}{$\mathrm{NA}_{2}$} \\
\hline & $\mathrm{RR}$ & $\mathrm{OM}$ & $\mathrm{AB}$ & ID & $\mathrm{IG}$ & $\mathrm{RR}$ & $\mathrm{OM}$ & $\mathrm{AB}$ & ID & $\mathrm{IG}$ \\
\hline & $R R$ & $O M$ & $A B$ & $S I$ & $L I$ & $R R$ & $O M$ & $A B$ & $S I$ & $L I$ \\
\hline 4760 & 86,23 & 98,50 & 98,36 & 99,77 & 99,94 & 84,09 & 96,95 & 99,06 & 99,41 & 99,75 \\
\hline 2380 & 83,35 & 94,74 & 93,52 & 96,95 & 98,29 & 80,15 & 94,26 & 95,99 & 97,13 & 97,90 \\
\hline 1190 & 77,71 & 91,26 & 83,72 & 94,16 & 96,27 & 73,35 & 91,14 & 87,58 & 94,06 & 96,01 \\
\hline 0590 & 64,99 & 78,22 & 66,47 & 78,66 & 84,28 & 59,41 & 77,13 & 73,79 & 81,31 & 83,39 \\
\hline 0297 & 43,04 & 54,70 & 44,38 & 55,32 & 60,07 & 38,27 & 53,44 & 51,00 & 59,29 & 60,28 \\
\hline 0149 & 31,24 & 28,64 & 22,86 & 29,08 & 31,71 & 18,54 & 28,12 & 26,69 & 31,82 & 33,38 \\
\hline
\end{tabular}

${ }_{1}^{1} \mathrm{RR}$ - rúmen-retículo; $\mathrm{OM}$ - omaso; $\mathrm{AB}$ - abomaso; ID - intestino delgado; IG - intestino grosso.

$1 R R$ - reticulum-rumen; OM - omasum; $A B$ - abomasum; SI - small intestine; $L I$ - large intestine.

passam preferencialmente pelo orifício retículoomasal. No caso do AB, 83,72 e $87,58 \%$ das partículas das digestas referentes aos tratamentos $\mathrm{NA}_{1} \mathrm{e}$ $\mathrm{NA}_{2}$, respectivamente, passaram pela peneira de $1,19 \mathrm{~mm}$. Esses valores são menores que os observados para OM, ID e IG, mas, conforme referido anteriormente, houve formação de agregados de partículas em parte das amostras da seção, após secagem em estufa, que não se desfizeram completamente com repetidas lavagens com acetona, e presença de sementes de braquiária, que influenciaram os resultados.

A presença de sementes de braquiária em amostras do $A B$, retidas na peneira de $1,19 \mathrm{~mm}$, sugere possível influência da densidade da referida partícula na passagem pelo orifício retículo-omasal. A influência da gravidade específica da partícula sobre sua probabilidade de deixar o RR, tem sido observada por vários autores (REID, 1984 e 1986; WAGHORN et al., 1986), segundo os quais partículas de alimento de menor tamanho e com maior densidade específica tendem a concentrar-se na região ventral do retículo, durante a abertura do orifício retículo-omasal, o que eleva sua chance de deixar o RR. McBRIDE et al. (1983), por sua vez, concluíram que apenas o tamanho não explica a maior probabilidade de partículas de $1 \mathrm{a}$ $2 \mathrm{~mm}$ deixarem o rúmen, conforme tem sido verificado por autores (ULYATT et al., 1986; SANCHES REYES, 1994), e conforme sugerem os resultados do presente trabalho, uma vez que o orifício retículoomasal tem abertura superior a $4 \mathrm{~mm}$.
Observou-se, ao abate, aumento do conteúdo de MS do RR com elevação do nível de consumo alimen$\operatorname{tar}(\mathrm{P}<0,05$, Tabela 4$)$, o que está de acordo com os resultados observados por OKINE e MATHISON (1991), ao compararem vacas não lactentes ingerindo alimento em quantidades crescentes, variando de 1 a 1,7 vezes a mantença.

O nível de ingestão alimentar não teve influência significativa sobre a taxa de desaparecimento da MS no RR embora se observasse ligeiro aumento em seu valor numérico, com a elevação do consumo. A taxa de desaparecimento corresponde à soma das taxas de passagem e de degradação e é calculada dividindo-se a ingestão de MS por hora, pelo conteúdo ruminal de MS. A elevação da taxa de consumo, sem aumento correspondente na taxa de desaparecimento, determinou elevação do conteúdo de MS ruminal. FRAZOLIN etal.(1995), estudando a cinética ruminal de forrageiras tropicais, encontrou taxas de degradação da MS de 5,0; 3,9 e 3,9\%/hora para os capins napier, setária e gordura, respectivamente.

A taxa de passagem da FDN total no RR, equivalente à taxa de desaparecimento ou de ingestão de FDNI, uma vez que a fração indigestível da FDN somente desaparece do rúmen pela passagem pelo orifício retículo-omasal, não se elevou $(\mathrm{P}>0,05)$ com o aumento do consumo, de maneira semelhante ao verificado para MS.

As taxas de passagem da FDN encontradas no presente trabalho foram relativamente altas, considerando-se que os níveis de consumo $\mathrm{NA}_{1}$ e $\mathrm{NA}_{2}$

Rev. bras. zootec., 30(6S):2134-2144, 2001 
corresponderam, aproximadamente, a 1,1 e 1,6 vezes a mantença. Taxas de passagem da FDN igualmente elevadas, para forrageiras tropicais, de 4,84\%/hora no verão e 3,59\%/hora no inverno foram observadas por ALMEIDA (1997) em novilhos mestiços Holandês-Zebu, com peso de 350 a $400 \mathrm{~kg}$, mantidos em pasto, contendo diferentes espécies de forrageiras tropicais, características da Zona da Mata de Minas Gerais. As altas taxas de passagem observadas no presente trabalho podem ser atribuídas à qualidade do pasto ingerido, uma vez que os animais foram mantidos em pastejo rotacionado em capim-elefante, com período de descanso de 32 dias, tendo, portanto, acesso à forragem de fácil degradabilidade, com possibilidade de selecionar o alimento ingerido. Isto resultou em baixo conteúdo ruminal de MS, mesmo nos animais do tratamento $\mathrm{NA}_{2}$, que, embora consumissem, em média, 7,98 $\mathrm{kg}$ de MS por dia, tiveram conteúdo ruminal de apenas $5,67 \mathrm{~kg}$ de MS.

Os tempos médios de retenção \pm erro-padrão da FDN no RR para os tratamentos $\mathrm{NA}_{1}$ e $\mathrm{NA}_{2}$,
$27,42 \pm 2,2$ e $26,64 \pm 2,2$ horas, respectivamente, não diferiram entre si $(\mathrm{P}>0,05)$. De forma diferente, OKINE e MATHISON (1991) verificaram redução do tempo de retenção com elevação do consumo. Para forrageiras tropicais, ALMEIDA (1997) encontrou tempos de retenção da fase sólida da digesta ruminal, em novilhos consumindo pasto contendo várias espécies forrageiras de 21,15 e 28,07 horas, no verão e inverno, respectivamente.

O nível de ingestão de forragem não teve efeito sobre a taxa de desaparecimento da FDN, no rúmen $(\mathrm{P}>0,05)$, indicando ter ocorrido aumento no seu conteúdo ruminal proporcionalmente semelhante ao aumento do consumo, nos animais do tratamento $\mathrm{NA}_{2}$.

O nível de ingestão alimentar não afetou $(\mathrm{P}>0,05)$ a taxa de degradação ruminal da FDN total $(2,46 \pm 0,23$ e $3,12 \pm 0,23 \%$ /hora, respectivamente, para os tratamentos $\mathrm{NA}_{1}$ e NA $\mathrm{NA}_{2}$, o que concorda com os resultados de OKINE e MATHISON (1991), que não verificaram diferença na taxa de degradação da FDN

Tabela 4 - Valores médios \pm erro-padrão referentes a variáveis ligadas à cinética ruminal em novilhos, para dois níveis de ingestão de pasto de capim-elefante: $\mathrm{NA}_{1}$ - com restrição alimentar; $\mathrm{NA}_{2}$ - sem restrição alimentar

Table 4 - Mean values \pm standard error for traits of ruminal kinetics in steers grazing elephantgrass under two levels of intake

\begin{tabular}{|c|c|c|}
\hline \multirow[t]{2}{*}{$\begin{array}{l}\text { Variável }^{1} \\
\text { Variable }^{1}\end{array}$} & \multicolumn{2}{|c|}{$\begin{array}{c}\text { Níveis de alimentação } \\
\text { Feedinglevel }\end{array}$} \\
\hline & $\mathrm{NA}_{1}$ & $\mathrm{NA}_{2}$ \\
\hline Consumo diário de matéria seca $(\mathrm{kg})$ & $5,41^{b_{ \pm}} \pm 0,25$ & $7,98^{\mathrm{a}} \pm 0,25$ \\
\hline Dry matter intake (kg) & & \\
\hline Conteúdo de MS no rumen-retículo & $4,27^{b_{ \pm}} 0,31$ & $5,67^{\mathrm{a}} \pm 0,31$ \\
\hline $\begin{array}{l}\text { Dry matter rumen pool } \\
\text { Taxa de desaparecimento da MS no RR }(\% / \mathrm{h})\end{array}$ & $5,28{ }^{a} \pm 0,37$ & $5,86^{\mathrm{a}} \pm 0,37$ \\
\hline Disappearance rate of DM in $R R(\% / h)$ & & \\
\hline $\begin{array}{l}\text { Conteúdo ruminal de FDN }(\mathrm{kg}) \\
\text { NDF rumen pool }\end{array}$ & $2,73^{b_{ \pm}} 0,21$ & $3,57^{a} \pm 0,21$ \\
\hline Taxa de passagem da FDN no RR $(\% / \mathrm{h})$ & $3,65^{\mathrm{a}} \pm 0,25$ & $3,75^{\mathrm{a}} \pm 0,25$ \\
\hline $\begin{array}{l}\text { Passage rate of } N D F \text { in } R R(\% / h) \\
\text { Taxa de desaparecimento da FDN total }(\% / \mathrm{h})\end{array}$ & $6,11 \pm 0,42$ & $6,87^{a} \pm 0,42$ \\
\hline Disappearance rate of total NDF $(\% / h)$ & & \\
\hline $\begin{array}{l}\text { Taxa de degradação da FDN total }(\% / \mathrm{h}) \\
\text { Digestion rate of total NDF }(\% / h)\end{array}$ & $2,46^{a_{1}} \pm 0,23$ & $3,12^{\mathrm{a}_{ \pm}} 0,23$ \\
\hline $\begin{array}{l}\text { Taxa de desaparecimento de FDN digestível }(\% / \mathrm{h}) \\
\text { Disappearance rate of digestible NDF }(\% / h)\end{array}$ & $14,38^{a} \pm 1,60$ & $17,76^{\mathrm{a}} \pm 1,60$ \\
\hline $\begin{array}{l}\text { Taxa de degradação da FDN digestível }(\% / \mathrm{h}) \\
\text { Digestion rate of digestible NDF }(\% / h)\end{array}$ & $10,73^{\mathrm{a}} \pm 1,59$ & $14,00^{\mathrm{a}} \pm 1,59$ \\
\hline $\begin{array}{l}\text { Digestibilidade aparente da FDN no RR }(\%) \\
\text { Apparent digestibility of NDF in } R R(\%)\end{array}$ & $44,22^{\mathrm{a}} \pm 1,60$ & $47,02^{\mathrm{a}} \pm 1,60$ \\
\hline $\begin{array}{l}\text { Tempo médio de retenção da FDN no RR (h) } \\
\text { Average retention time of NDF in } R R(h)\end{array}$ & $27,42^{a} \pm 2,22$ & $26,64^{\mathrm{a}} \pm 2,22$ \\
\hline $\begin{array}{l}\text { Tempo de renovação do RR }(\mathrm{h}) \\
\text { Turnover time of } R R(h)\end{array}$ & $18,94^{\mathrm{a}} \pm 1,09$ & $17,06^{a_{1}} \pm 1,09$ \\
\hline
\end{tabular}

Médias seguidas das mesmas letras minúsculas na linha não diferem, segundo o teste $F(P>0,05)$.

Means followed by the same small letter in the rows do not differ, by $F$ test $(P>.05)$.

Rev. bras. zootec., 30(6S):2134-2144, 2001 
total, à medida que o consumo se elevou no intervalo de 1 a 1,7 vezes a mantença, em animais alimentados com dietas contendo apenas volumosos. FRAZOLIN et al. (1995) encontraram para o capim-napier taxa de degradação de 4,7\%/hora.

Não houve diferença entre tratamentos quanto a taxa de desaparecimento da FDN digestível $(\mathrm{P}>0,05)$. $\mathrm{O}$ aumento do consumo diário de FDN digestível, no tratamento $\mathrm{NA}_{2}$, foi contrabalançado por aumento, em proporção semelhante, do conteúdo ruminal da citada fração, não ocorrendo mudança $(\mathrm{P}>0,05)$ nas taxas de passagem e de degradação da FDN digestível, com elevação do consumo.

O nível de ingestão alimentar não afetou $(\mathrm{P}>0,05)$ o tempo de renovação do conteúdo rumino-reticular. Do mesmo modo, não houve diferença entre tratamentos, quanto à digestibilidade aparente da FDN no rúmen $(\mathrm{P}>0,05)$.

\section{Conclusões}

Os maiores valores estimados para o módulo de finura, para o tamanho médio de partícula e para a proporção retida na peneira de maior abertura $(4,760 \mathrm{~mm})$, corresponderam às partículas da digesta rumino-reticular. Essas variáveis e as estimativas dos parâmetros da cinética de degradação e passagem da fibra, obtidas por meio da técnica da evacuação ruminal, não foram afetadas pelo nível de ingestão do pasto.

\section{Referências Bibliográficas}

ALMEIDA, M.S. Consumo voluntário e sua correlação com aspectos qualitativos e quantitativos da forragem. Viçosa, MG: UFV, 1997. 102p. Tese (Doutorado em Zootecnia) Universidade Federal de Viçosa, 1997.

AMERICAN DAIRY SCIENCE ASSOCIATION 1970. A report: commitee on classification of particle size in feedstuffs. J. Dairy. Sci., 53:689-690.

AMERICAN SOCIETY OF AGRICULTURAL ENGINEERS 1969a. ASAE standard: ASAE s.319. Method of determining and expressing fineness offeed materials by sieving. Agric. Eng. Yearb., St. Joseph, Mich, U.S. p.346-347.

AMERICAN SOCIETY OF AGRICULTURAL ENGINEERS 1969b. ASAE Recommendation: ASAE R 24b. Method of determinning modulus of uniformity and modulus of fineness of groudfeed. Agric. Eng. Yearb., St. Joseph, Mich., U. S., p.348.

AMERICAN SOCIETY OF ANIMAL SCIENCE. 1969. Summary of the minutes of the 61 st. Business meeting of the American Society of Animal Science. J. Anim. Sci., 29:1021.

BOSCH, M.W., BRUINING, M. 1995. Passage rate and total clearance rate from the rumen of cows feed. on grass silages differing in cell-wall content. Brit. J. Nutr., 73:41-49.
DADO, R.G., ALLEN, M.S. 1995. Intake limitations, feeding behavior, and rumen function of cows challenged with rumen fill from dietary fiber or inert bulk. J. Dairy Sci., 78: 118-133.

ENSOR, W.L., OLSON, H.H., COLENBRANDER, V.F. 1970. A report: Committee on classification of particle size in feedstuffs. J. Dairy Sci., 53:689-690.

FAICHNEY, G.J. 1986. The kinetcs of particule matter in the rumen. In: MILLIGAN, L. P., GROVUM, W. L., DOBSON, A. (Eds.) Control of digestion and metabolism in ruminants. Englewood Cliffs, Prentice-hall. p.173-195.

FISCHER, D.S., BURNS, J.C., POND, K.R. 1988. Estimation of mean and median particle size of ruminant digesta. J. Dairy Sci., 71:518-524.

FRANZOLIN, R., HERLING, V.R., NOGUEIRA FILHO, J.C.M. 1995. Degradabilidade in situ de gramíneas e leguminosas em búfalos sob pastejo. R. Soc. Bras. Zootec., 24(1):8-19.

FREITAS, S.P. Características químicas e físicas da digesta de bezerros provenientes de rebanhos leiteiros recebendo diferentes niveis de concentrado.Viçosa, MG: UFV, 1997.68p. Dissertação (Mestrado em Zootecnia)-Universidade Federal de Viçosa, 1997.

LEE, J.A., PEARCE, G.R. 1984. The effectiveness of chewing during eating on particle size reduction of roughages by cattle. Austr. J. Agric. Res., 35:609-618.

LUNGINBUHL, J.M., POND, K.R., BURNS, J.C. 1990. Changes in ruminal and fecal particle weight distribution of steers fed. coastal bermudagrass hay at four levels. J. Anim. Sci., 68:2864-2873.

MARTZ, F.A., BELYEA, R.L. 1986. Role of particle size and forage quality in digestion and passage by cattle and sheep. J. Dairy Sci., 69:1996-2008.

McBRIDE, B.W., MILliGAN, L.P., TURNER, B.V. 1983. Endoscopic observation of the reticulo-omasal orifice of cattle. J. Agric. Sci., 101:749-750.

McLEOD, M.N., MINSON, D.J. 1988a. Large particle breakdown by cattle eating ryegrass and alfalfa.J. Anim. Sci., 66(9):992-999.

OKINE, E.K., MATHISON, G.W. 1991. Effects of feed intake on particle distribution, passage of digesta, and extent of digestion inthe gastrointestinal tract of cattle. J. Anim. Sci., 69: 3435-3445.

OSBOURN, D.F., BEEVER, D.E., THOMSON, D.J. 1976. Influence of physical processing on the intake, digestion and utilization of dried herbage. Proc. Nutr. Soc., 35:191.

POPPI, D.P., HENDRICKSEN, R.E., MINSON, D.J. 1985. The relative resistance to escape of leaf and stem particle from the rumen of cattle and sheep. J. Agric. Sci., 105:9-14.

POPPI, D.P., MINSON, D.J., TERNOUTH, J.H. 1981b. Studies of cattle and sheep eating leaf and stem fractions of grasses. II. Factors controlling the retention of feed in the reticulorumen. Austr. J. Agric. Res., 32:109-122.

POPPI, D.P., NORTON, B.W., MINSON, D.J. et al. 1980. The validity of the critical size theory for particles leaving the rumen. J. Agric. Sci., 94:257-280.

QUEIROZ, A.C., SANCHEZ, N.R., RESENDE, F.D. et al. 1996a. Tamanho médio de partícula, módulos de finura e de uniformidade da digesta ao longo do trato gastrintestinal de ruminantes de diferentes grupos genéticos. R. Soc. Bras. Zootec., 25(1):125-141.

REID, C.S.W. 1986. Digestive physiology: The challenges today and tomorrow. In: MILLIGAN, L.P., GROVUM, W.L., DOBSON, A. (Eds.) Control of digestion and metabolism in ruminants. Reston, Reston Publishing. p.540-557. 
REID, C.S.W. 1984. The progress of solid feed redidues through the rumino-reticulum: the ins and outs of particles. In: BAKER, S.K., GAWTHORNE, J.M., MACKINTOSH, J.B. et al. (Eds.) Ruminant phisiology concepts and consequences. Murdoch, University Western Australian, Nedlands. p.79-84.

SANCHEZ REYES, N. Concentração de nutrientes e distribuição de partículas na digesta, em ruminantes de diferentes grupos raciais. Viçosa, MG: UFV, 1994. 98p. Dissertação (Mestrado em Zootecnia)-Universidade Federal de Viçosa, 1994.

SHAVER, R.D., SATTER, L.D., JORGENSEN, N.A. 1988. Impact of forage fiber content on digestion and digesta passage in lactating dairy cows. J. Dairy Sci., 71:1556-1565.

SMITH, L.W., WALDO, D.R. 1969. Method for sizing forage cell wall particles. J. Dairy Sci., 52:2051-2053.

STENSIG, T., WEISBJERG, M.R., HVELPLUND, T. 1998. Evaluation of different methods. Acta Agric. Scand., Sect. A, Anim. Sci., 48:141-154.

SUTHERLAND, T.M. 1988. particle separation in the forestomach of sheep. In: DOBSON, A., DOBSON, M.J. (Eds.) Aspects of digestive physiology in ruminants. Ithaca, Ithaca Comstock Publishing Associates. p.43-47.

TILLEY, J.M., TERRY, R.A. 1963. A two-stage technique for the in vitro digestion of forage crops. J. Br. Gr. Sci., 18(2):104-11.
ULYATT, M.J., DELLOW, D.W., JOHN, A., REID, C.S.W., WAGHORN, G.S. 1986. The contribution of chewing during eating and rumination to the clearance of digesta from the rumino-reticulum. In: MILLINGAN, L.P., GROVUM, W.L., DOBSON, A. (Eds.) Control of digestion and metabolism in ruminants. Englewood Cliffs, Prentice hall. p.498-515.

VAN SOEST, P.J., SNIFFEN, C.J., ALLEN, M.S. 1988. Rumen dynamics. In: DOBSON, A., DOBSON, M.J. (Eds.) Aspects of digestive physiology in ruminants. Ithaca, Cornell University Press. p.21-42.

WAGHORN, G.C., REID, C.S.W., ULYATT, M.J. et al. 1986. Feed comminution, particle composition and distribution between a the four compartments of the stomach in sheep feed. chopped lucerne hay at two feeding frequencies and intake level. J. Agric. Sci., 106:287-296.

WALDO, D.R., SMITH, L.W., COX, E.L. 1972. Model of cellulose disappearance from the rumen. J. Dairy Sci., 55(1):125-129.

Recebido em: 29/05/00

Aceito em: 11/06/01 